Research Article

\title{
Trajectory Tracking of a Flexible Robot Manipulator by a New Optimized Fuzzy Adaptive Sliding Mode-Based Feedback Linearization Controller
}

\author{
M. J. Mahmoodabadi iD and N. Nejadkourki \\ Department of Mechanical Engineering, Sirjan University of Technology, Sirjan, Iran \\ Correspondence should be addressed to M. J. Mahmoodabadi; mahmoodabadi@guilan.ac.ir
}

Received 19 March 2020; Revised 25 May 2020; Accepted 2 June 2020; Published 10 July 2020

Academic Editor: Weitian Wang

Copyright (c) 2020 M. J. Mahmoodabadi and N. Nejadkourki. This is an open access article distributed under the Creative Commons Attribution License, which permits unrestricted use, distribution, and reproduction in any medium, provided the original work is properly cited.

\begin{abstract}
This work presents a novel fuzzy adaptive sliding mode-based feedback linearization controller for trajectory tracking of a flexible robot manipulator. To reach this goal, after deriving the dynamical equations of the robot, the feedback linearization approach is utilized to change the nonlinear dynamics to a linear one and find the control law. Then, the sliding mode control strategy is implemented to design a stabilizer for trajectory tracking of the flexible robot. In order to adaptively tune the parameters of the designed controller, the gradient descent approach and the chain derivative rule are employed. Moreover, the Takagi-Sugeno-Kang fuzzy system is applied to regulate the controller gains. Finally, a multiobjective particle swarm optimization algorithm is used to find the optimum fuzzy rules. The conflicting objective functions considered as the integrals of the absolute values of the state error and the control effort should be minimized, simultaneously. The simulation results illustrate the effectiveness and capability of the introduced scenario in comparison with other methods.
\end{abstract}

\section{Introduction}

In the recent years, the study of the flexible robots has been widely developed. The main reasons for this attraction could be mentioned as reaching to exact solutions and accurate performances. In fact, in order to reach high stiffness for rigid manipulators, their volume and weight would be increasingly raised, and therefore, the performance speed and energy consumption might be incremented [1-5].

On the contrary, all actual systems are inherently nonlinear, and their governing dynamical equations are also nonlinear. For stabilization of these systems, two approaches have been commonly proposed. The first method is based on the linearization about the equilibrium point via Taylor expansion and named the Jacobian method. The main limitations of this scheme are neglecting the nonlinearities and operating around the equilibrium point. The second one is the feedback linearization method that changes the nonlinear variables instead of neglecting them [6-10].
After changing the variables via the feedback linearization method, it would be better to apply a nonlinear controller to stabilize the system instead of a linear one. In this work, the sliding mode control, initially introduced by Utkin in 1997 [11] as a robust, powerful, and nonlinear approach, is utilized for the considered flexible robot manipulator. After that, a lot of researchers have applied this approach in many fields of science and technology such as Xiong et al. solved the distributed sliding mode control problem of complex networks with inner coupling under a quantization mechanism [12]. Yuguang and Fan proposed a method for dynamic modeling and a modified sliding mode control for multilink underwater manipulators [13]. An adaptive funnel sliding mode controller for servo mechanisms with friction compensation was introduced by Wang et al. [14]. Divandari et al. suggested the fuzzy fast terminal sliding mode control method for speed control of a switched reluctance motor [15]. A voltage-based sliding mode control was presented to control the position of the serial robot 
manipulators by Zaare et al. [16]. Peza-Solís et al. intended modeling a single flexible-link robot using the finite difference method and sliding mode control [17]. Two different sliding mode control approaches for the trajectory control of a flexible-link robot were investigated in the theory, simulation, and experiments by Hisseine and Lohmann [18].

Moreover, one of the main issues for the controller design is the appropriate regulation of the gains. The most common schemes to tune the control parameters are adaptation approaches that have been widely utilized to improve the performance of the controllers. To name but a few, Miao et al. recommended an adaptive fast nonsingular terminal sliding mode control scheme for a spacecraft with a rotating flexible appendage with accurate attitude tracking [19]. Zhao et al. concerned the vibration control and global stabilization of a distributed parameter flexible-riser system in the presence of the nonlinear input saturation and system uncertainties by an adaptive robust method [20]. An effective model-free adaptive fault-tolerant attitude-tracking controller was developed for a flexible spacecraft with flexible solar arrays actuated using redundant reaction wheels in the presence of inertia uncertainties, external disturbances, and uncertain actuator failures by Boulouma et al. [21]. Gierlak and Szuster presented an approach to control an object operating with a flexible environment based on the knowledge of the mathematical structure of the system model [22]. A systematic adaptive control approach was investigated by Seyed Danesh et al. to achieve the prediction of optimal input displacement for a gripper by means of an extreme learning machine strategy [23].

Besides, fuzzy systems based on the fuzzy logic are the only way to employ the human knowledge and linguistic variables for modeling the dynamical systems. For instance, Zheng et al. suggested an intelligent fuzzy sliding mode control method that takes the advantages of the sliding mode control, fuzzy control, and deep learning algorithms and successfully applied on a robot [24]. A prescribed performance tracking control scheme was presented by Shi for a class of multi-input multioutput nonlinear systems having immeasurable states and unknown control direction [25]. Hongqian et al. awarded the circle criterion and its extended version to analyze the global asymptotical stability of the simplest Takagi-Sugeno fuzzy control system in the frequency domain [26]. Self-tuning based on the evolutionary algorithms for the first-order Takagi-Sugeno-Kang-type fuzzy controller was applied for the trajectory tracking of a quadcopter drone by Yazid et al. [27].

Finally, one of the most common and well-known ways to determine the appropriate values for the constant parameters of the controller is implementation of the evolutionary optimization algorithms. These methods, usually inspired by a natural phenomenon, have been widely developed to solve different scientific problems in the recent decades: to name but a few, particle swarm optimization [28-30], firefly optimization algorithm [31], ant colony optimization algorithm [32-35], genetic algorithm [36-39], imperialist competitive algorithm [40-42], team game algorithm [43], and teacher-learning-optimization algorithm $[44,45]$. On the contrary, most of engineering problems, especially controller design, have more than one objective function (criterion) for optimization [46-50]. As a success approach, multiobjective high exploration particle swarm optimization is a recently introduced algorithm by the authors of this work to solve real-world and complicated multicriterion problems [51]. This algorithm utilizes the main operators of three schemes, the particle swarm optimization, the bee colony algorithm, and the multi-crossover genetic algorithm, to enhance the quality of solutions and guide them to the global best position [52].

The motivation of this research is to design a novel combination of the fuzzy logic, adaptation laws, and sliding mode concepts with the feedback linearization approach and the multiobjective particle swarm optimization. In fact, at first, the dynamical equations of a flexible robot are derived and linearized by the feedback linearization method. Then, the robust and nonlinear sliding mode scheme is successfully applied to control the system states from the initial conditions to the desired values. The controller gains are tuned, regulated, and optimized by the gradient descent method, the fuzzy systems, and the PSO algorithm, respectively. The simulation results are illustrated to prove the ability, effectiveness, and efficiency of the introduced scenario to track the defined trajectory for the considered manipulator.

The remainder of the paper is arranged as follows. Section 2 presents the dynamical equations for the desired robot. Section 3 describes the linearization of the system using the feedback method. Section 4 describes the corresponding equations to the sliding mode control. Sections 5 and 6 , respectively, deal with the adaptation laws and the fuzzy system designed to improve the controller performance. Moreover, the optimization method, design variables, and objective functions are presented in Section 7. Section 8 presents simulation results and comparative studies to confirm the capability of the proposed method. Finally, Section 9 concludes the paper and describes the related future works.

\section{Dynamical Equations of the Flexible Robot}

The regarded single-link robot is shown in Figure 1, and its dynamical equations obtained by the Lagrange approach are written in the following relations [53]:

$$
J \ddot{\theta}_{2}-K\left(\theta_{1}-\theta_{2}\right)=v, I \ddot{\theta}_{1}+M g L \sin \left(\theta_{1}\right)+K\left(\theta_{1}-\theta_{2}\right)=0 \text {, }
$$

where $\theta_{1}$ and $\theta_{2}$ denote the angular positions of the motor shaft and the flexible link, respectively, $M, L$, and $I$, respectively, present the mass, the length, and the inertia moment of the link, $g$ is the gravity acceleration, $K$ is the stiffness of the flexible part of the link, $J$ is the inertia moment of the motor shaft, and, finally, $v$ shows the torque applied by the motor. If the state variables are defined as follows, 


$$
\begin{aligned}
& \xi_{1}=\theta_{1}, \\
& \xi_{2}=\dot{\theta}_{1}, \\
& \xi_{3}=\theta_{2}, \\
& \xi_{4}=\dot{\theta}_{2},
\end{aligned}
$$

then the state-space equations would be represented by the following relations:

$$
\begin{aligned}
& \dot{\xi}_{1}=\xi_{2}, \\
& \dot{\xi}_{2}=\frac{-M g L}{I} \sin \left(\xi_{1}\right)-\frac{K}{I}\left(\xi_{1}-\xi_{3}\right), \\
& \dot{\xi}_{3}=\xi_{4}, \\
& \dot{\xi}_{4}=\frac{K}{J}\left(\xi_{1}-\xi_{3}\right)+\frac{1}{J} v .
\end{aligned}
$$

Hence, the matrix form of the governing equations could be stated as follows:

$$
\dot{\xi}=h_{1}(\xi)+h_{2}(\xi) v,
$$

where

$$
h_{1}(\xi)=\left[\begin{array}{c}
\xi_{2} \\
\frac{-M g L}{I} \sin \left(\xi_{1}\right)-\frac{K}{I}\left(\xi_{1}-\xi_{3}\right) \\
\xi_{4} \\
\frac{K}{J}\left(\xi_{1}-\xi_{3}\right) \\
h_{2}(\xi)=\left[\begin{array}{c}
0 \\
0 \\
0 \\
\frac{1}{J}
\end{array}\right] .
\end{array}\right.
$$

\section{Feedback Linearization}

As it can be seen from the dynamical equations of the system represented in the previous section, the order of the system is $n=4$. Therefore, the necessary and sufficient conditions for feedback linearization of this system could be mentioned as follows:

$$
\operatorname{rank}\left[h_{2}, a d_{h_{1}}\left(h_{2}\right), a d_{h_{1}}^{2}\left(h_{2}\right), a d_{h_{1}}^{3}\left(h_{2}\right)\right]=4,
$$

and that the set

$$
\left\{h_{2}, a d_{h_{1}}\left(h_{2}\right), a d_{h_{1}}^{2}\left(h_{2}\right)\right\}
$$

be involute. Regarding the indicated conditions, it is easy to check that

$$
\left[h_{2}, a d_{h_{1}}\left(h_{2}\right), a d_{h_{1}}^{2}\left(h_{2}\right), a d_{h_{1}}^{3}\left(h_{2}\right)\right]=\left[\begin{array}{cccc}
0 & 0 & 0 & \frac{K}{I J} \\
0 & 0 & \frac{K}{I J} & 0 \\
0 & \frac{1}{J} & 0 & \frac{-K}{J^{2}} \\
\frac{1}{J} & 0 & -\frac{K}{J^{2}} & 0
\end{array}\right] \text {, }
$$

which has rank 4 for $K>0$ and $I, J<\infty$. Moreover, since fields $\left\{h_{2}, a d_{h_{1}}\left(h_{2}\right), a d_{h_{1}}^{2}\left(h_{2}\right)\right\}$ are constant, they form an involutive set. To see this, it is sufficient to note that the Lie bracket of two constant vector fields is zero. Hence, the Lie bracket of any two members of the set of the vector field in equation (7) is zero as a linear combination of the regarded vector fields $[6,7]$. It follows that the system illustrated as equation (4) is feedback-linearizable. Therefore, the new coordinates are defined as follows based upon $n=4$ :

$$
z_{i}=\tau_{i}, \quad i=1, \ldots, 4,
$$

where

$$
\begin{aligned}
L_{h_{2}} \tau_{1} & =0, \\
L_{\left\{h_{1}, h_{2}\right\}} \tau_{1} & =0, \\
L_{a d_{h_{1}}^{2} h_{2}} \tau_{1} & =0, \\
L_{a d_{h_{1}}^{3} h_{2}} \tau_{1} & =0 .
\end{aligned}
$$

Carrying out the above calculations leads to the following set of equations:

$$
\begin{aligned}
& \frac{\partial \tau_{1}}{\partial \xi_{2}}=0, \\
& \frac{\partial \tau_{1}}{\partial \xi_{3}}=0, \\
& \frac{\partial \tau_{1}}{\partial \xi_{4}}=0, \\
& \frac{\partial \tau_{1}}{\partial \xi_{1}} \neq 0 .
\end{aligned}
$$

It is obvious that variable $\tau_{1}$ is only dependent to $\xi_{1}$. Therefore, a simple solution for $\tau_{1}$ could be mentioned as follows:

$$
z_{1}=\tau_{1}=\xi_{1}
$$

The other variables are obtained as follows: 


$$
\begin{aligned}
& z_{2}=\tau_{2}=L_{h_{1}} \tau_{1}=\xi_{2}, \\
& z_{3}=\tau_{3}=L_{h_{1}} \tau_{2}=\frac{-M g L}{I} \sin \left(\xi_{1}\right)-\frac{K}{I}\left(\xi_{1}-\xi_{3}\right), \\
& z_{4}=\tau_{4}=L_{h_{1}} \tau_{3}=\frac{-M g L}{I} \cos \left(\xi_{1}\right)-\frac{K}{I}\left(\xi_{2}-\xi_{4}\right) .
\end{aligned}
$$

The following control input is defined to linearize the above system:

$$
v=\frac{1}{L_{h_{2}} \tau_{4}}\left(u-L_{h_{1}} \tau_{4}\right) .
$$

Therefore,

$$
\begin{aligned}
v= & \frac{I J}{K}(u-a(\xi))=\beta(\xi) u+\alpha(\xi), \\
a(\xi)= & \frac{M g L}{I} \sin \left(\xi_{1}\right)\left(\xi_{2}^{2}+\frac{M g L}{I} \cos \left(\xi_{1}\right)+\frac{K}{I}\right) \\
& +\frac{K}{I}\left(\xi_{1}-\xi_{3}\right)\left(\frac{K}{I}+\frac{K}{J}+\frac{M g L}{I} \cos \left(\xi_{1}\right)\right) .
\end{aligned}
$$

Based on new coordinates $\left(z_{1}, \ldots, z_{4}\right)$ and by applying the control law (18), the governing equations of the system could be represented as follows:

$$
\begin{aligned}
& \dot{z}_{1}=z_{2}, \\
& \dot{z}_{2}=z_{3}, \\
& \dot{z}_{3}=z_{4}, \\
& \dot{z}_{4}=u .
\end{aligned}
$$

These relations could be mentioned in the matrix form as follows:

$$
z=\Xi \dot{z}+\varrho u
$$

where

$$
\begin{aligned}
& \Xi=\left[\begin{array}{llll}
0 & 1 & 0 & 0 \\
0 & 0 & 1 & 0 \\
0 & 0 & 0 & 1 \\
0 & 0 & 0 & 0
\end{array}\right], \\
& \varrho=\left[\begin{array}{l}
0 \\
0 \\
0 \\
1
\end{array}\right] .
\end{aligned}
$$

By considering equations (13)-(15), the main state variables of the flexible robot are obtained as follows:

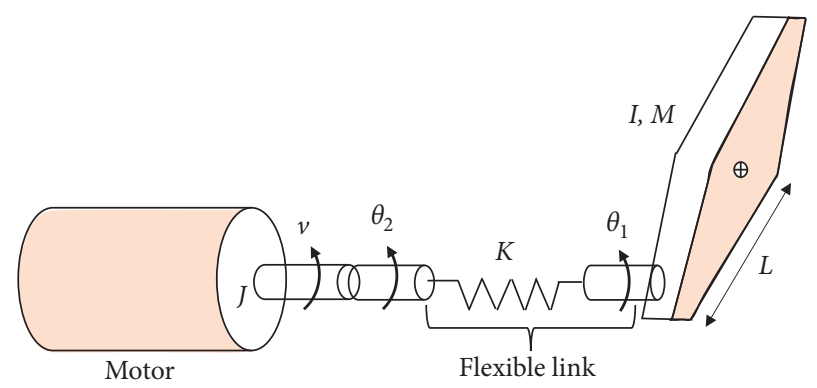

FIgURE 1: Single-link flexible-joint robot and the related parameters.

$$
\begin{aligned}
& \xi_{1}=z_{1}, \\
& \xi_{2}=z_{2}, \\
& \xi_{3}=z_{1}+\frac{I}{K}\left(z_{3}+\frac{M g L}{I} \sin \left(z_{1}\right)\right), \\
& \xi_{4}=z_{2}+\frac{I}{K}\left(z_{4}+\frac{M g L}{I} z_{2} \cos \left(z_{1}\right)\right) .
\end{aligned}
$$

\section{Sliding Mode Control}

Sliding control is a powerful approach for stabilization of nonlinear systems in the presence of modeling uncertainties and parametric disturbances $[54,55]$. This scheme is based on the idea that controlling a first-order system is much easier than a general $n^{\text {th }}$-order one. Usually, the sliding mode scalar function $\psi$ is defined as follows:

$$
\begin{aligned}
\psi= & \left(\frac{\mathrm{d}}{\mathrm{d} t}+\delta\right){ }^{n-1} e=\left(\begin{array}{c}
n-1 \\
0
\end{array}\right) e^{(n-1)}+\left(\begin{array}{c}
n-1 \\
1
\end{array}\right) \delta e^{(n-2)} \\
& +\ldots+\left(\begin{array}{c}
n-1 \\
n-1
\end{array}\right) \delta^{n-1} e,
\end{aligned}
$$

where $e$ is the tracking error vector, $\delta$ is a positive parameter, and binomial coefficient $\left(\begin{array}{l}n \\ k\end{array}\right)$ is defined as $n ! / k !(n-k)$ !. Equation $\psi=0$ defines a time-dependent surface in the space $R^{n}$, and $e(t)=0$ is its unique solution. Hence, the problem of tracking control will be equivalent to putting zero in the scalar function $\psi$. Based on the Lyapunov theory, control effort $v$ must be selected so that the following inequality is satisfied:

$$
\frac{1}{2} \frac{\mathrm{d}}{\mathrm{d} t} \psi^{2} \leq-\eta|\psi|,
$$

where $\eta$ is a positive constant.

On the contrary, the sliding mode controller commonly includes two parts: the equivalent term $\left(u_{e q}\right)$ and the switching control law $(\sigma \operatorname{sign}(\psi))$. The switching control law tries to guide the system states toward the sliding surface, and the equivalent control law, which is gained from solving the equation $\dot{\psi}=0$, guarantees that the system states stay on 
the sliding surface and converge to zero along the sliding surface.

$$
u=u_{e q}+\sigma \operatorname{sign}(\psi)
$$

where parameter $\sigma$ is selected so that Lyapunov condition (24) is satisfied. For a more uniform performance, saturation function $\operatorname{sat}(\psi / \varnothing)$, defined as follows, is replaced by function $\operatorname{sgn}(\psi)$ in equation (24):

$$
\operatorname{sat}\left(\frac{\psi}{\varnothing}\right)=\left\{\begin{array}{cc}
-1 & \text { if } \frac{\psi}{\varnothing} \leq-1 \\
\frac{\psi}{\varnothing} & \text { if } 1 \leq \frac{\psi}{\varnothing} \leq-1 \\
1 & \text { if } \frac{\psi}{\varnothing} \geq+1
\end{array}\right\},
$$

where $\varnothing$ is a positive parameter to illustrate the width of the boundary layer around the sliding surface. Considering the control problem of the flexible robot manipulator, the desired trajectory that must be tracked is defined as $z_{d}(t)=\sin (t)$, and the error signal will be $e=z_{1}-z_{d}$. Hence, the sliding surface is defined as follows:

$$
\psi=\left(\frac{\mathrm{d}}{\mathrm{d} t}+\delta\right)^{3} e=e^{(3)}+3 \delta e^{(2)}+3 \delta^{2} e^{(1)}+\delta^{3} e .
$$

Therefore,

$$
\begin{aligned}
\dot{\psi}= & e^{(4)}+3 \delta e^{(3)}+3 \delta^{2} e^{(2)}+\delta^{3} e^{(1)}=\dot{z}_{4}-z_{d}^{(4)}+3 \delta e^{(3)} \\
& +3 \delta^{2} e^{(2)}+\delta^{3} e^{(1)}=a^{\prime}+b^{\prime} u .
\end{aligned}
$$

Finally, by applying the sliding mode control theory, the control law can be stated as follows:

$$
\begin{aligned}
& u=-\frac{a^{\prime}}{b^{\prime}}+\sigma \operatorname{sat}\left(\frac{\psi}{\varnothing}\right) \\
& a \prime=-z_{d}^{(4)}+3 \delta e^{(3)}+3 \delta^{2} e^{(2)}+\delta^{3} e^{(1)}, \\
& b^{\prime}=1 .
\end{aligned}
$$

\section{Adaptation Laws}

The adaptation laws help to find the controller gains as dynamic and time varying so as the tracking procedure would be modified [56-60]. Furthermore, the adaptation laws used in this paper apply the robust sliding mode concepts to produce the time-varying functions for the control gains $(\sigma, \delta$, and as follows:

$$
\begin{gathered}
\widetilde{\sigma}=\sigma+\widehat{\sigma}, \\
\widetilde{\delta}=\delta+\widehat{\delta}, \\
\widetilde{\varnothing}=\varnothing+\widehat{\varnothing},
\end{gathered}
$$

where $\widetilde{\sigma}, \widetilde{\delta}$, and $\widetilde{\varnothing}$ are the tuned forms of control gains $\sigma, \delta$, and $\varnothing$. Moreover, $\widehat{\sigma}, \widehat{\delta}$, and $\widehat{\varnothing}$ are the adaptive parameters obtained via the gradient descent method as follows:

$$
\begin{aligned}
& \dot{\hat{\sigma}}=-\gamma_{1} \psi e, \\
& \dot{\widehat{\delta}}=-\gamma_{2} \psi e, \\
& \dot{\hat{\varnothing}}=-\gamma_{3} \psi e,
\end{aligned}
$$

where $\gamma_{1}, \gamma_{2}$, and $\gamma_{3}$ are named as the learning rate parameters that would be determined by the fuzzy systems introduced in the next section.

\section{Fuzzy Systems}

The fuzzy logic has been widely utilized to employ the human knowledge for system modeling. There are three types of fuzzy systems that are commonly used in the literature: (1) pure fuzzy systems, (2) Takagi-Sugeno-Kang (TSK) fuzzy systems, and (3) fuzzy systems with the fuzzifier and defuzzifier.

In this research, the Takagi-Sugeno-Kang (TSK) fuzzy system [61-65] is utilized for the regulation of learning rate parameters $\gamma_{1}, \gamma_{2}$, and $\gamma_{3}$ which could be constructed from the following rules: if $e$ is $A^{l}$, then $y_{j}^{l}=B_{j}^{l} e$, where $A^{l}(l=$ $1,2,3)$ are the fuzzy sets depicted in Figure $2, B_{j}^{l}$ are constant parameters for the $l$ th rule and $j$ th learning rate which would be found by the optimization process. Given an input $e \in U \subset \mathbb{R}$, output $\gamma_{j} \in V \subset \mathbb{R}$ of the TSK fuzzy system is computed as the weighted average of $y_{j}^{l_{s}} s$ as follows:

$$
\gamma_{j}=\frac{\sum_{L=1}^{3} y_{j}^{l} \mu_{A^{l}}}{\sum_{L=1}^{3} \mu_{A^{l}}}, \quad j=1,2,3,
$$

where, as it can be evident from the above equations, the TSK fuzzy system is a mapping from $U \subset \mathbb{R}$ to $V \subset \mathbb{R}$.

\section{Multiobjective Particle Swarm Optimization}

The particle swarm optimization (PSO) algorithm was initially proposed by Eberhart and Kennedy [66] in 1995 and inspired by the social behavior of birds. The PSO regards the search space of the problem as a flight space and considered each bird as a candidate solution for the problem. The basic idea of the algorithm is to find the optimal solution through cooperation and information sharing among the particles in the swarm.

In the PSO, each particle has its own position and velocity vectors, where the position of each particle is a potential solution. The personal historical best position of a particle $\left(\vec{p}_{\text {best }_{i}}\right)$ and the global best position of the swarm $\left(\vec{g}_{\text {best }}\right)$ are employed to guide the particle's flight and update its position as follows:

$$
\begin{aligned}
& \vec{s}_{i}(i t+1)= \alpha_{1} \vec{s}_{i}(i t)+\alpha_{2} \vec{\rho}_{1}\left(\vec{p}_{\text {best }_{i}}(i t)-\vec{p}_{i}(i t)\right) \\
&+\alpha_{3} \vec{\rho}_{2}(\vec{g} \text { best } \\
&\left.\vec{p}_{i}(i t)-\vec{p}_{i}(i t)\right), \\
&
\end{aligned}
$$




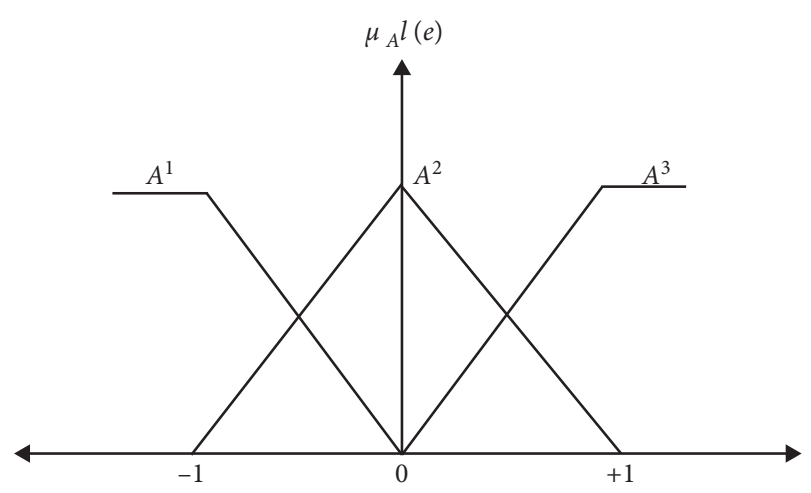

FIGURE 2: Input membership functions for the designed TSK fuzzy system.

where $\vec{p}_{i}(t)$ and $\vec{s}_{i}(t)$ are the position and velocity vectors of the $i$ th particle at the $i t$ th iteration, respectively. $\vec{\rho}_{1}$ and $\vec{\rho}_{2}$ symbolize the random vectors between $[0,1]$. Furthermore, $\alpha_{1}, \alpha_{2}$, and $\alpha_{3}$ denote the inertia parameter, cognitive coefficient, and social coefficient, respectively [66].

On the contrary, a multiobjective optimization problem has multiple conflicting objectives which need to be optimized, simultaneously. Unlike a single-objective optimization problem, the solution of the multiobjective optimization problem is not unique, and a set of the optimum solutions called Pareto front or nondominated solutions would be obtained and stored in external memory (archive) [51]. In most of multiobjective optimization methods, the archive contains a specified number of the optimum solutions having a good spread. However, if all nondominated solutions are maintained in the archive, then its size grows very quickly. In the regarded multiobjective algorithm, a fuzzy elimination technique is applied to prune the archive. In this approach, all particles in the archive have a neighborhood radius which is equal to $r_{F}$ (equation (34)), and if their Euclidean distances are fewer than $r_{F}$, then one of them would be randomly removed.

$$
r_{F}=\frac{i t}{\max i t} \times \frac{F V}{P C V}
$$

where it is the current iteration, max it is the maximum number of iterations, $P C V$ represents a positive constant value, and $F V$ denotes the fuzzy variable introduced in [51].

In order to assign a leader for the swarm in each iteration, a neighborhood method is suggested in the considered multiobjective particle swarm optimization algorithm. In this scheme, a neighborhood radius is defined for the archive members. If the Euclidean distance between two nondominated solutions is smaller than this radius, then they would be regarded as the neighbor. Finally, the nondominated solution having the fewer number of neighbors is assigned as the leader of the swarm [51]. The flowchart related to this procedure is depicted in Figure 3.

In this research, the constant control gains $(\sigma, \delta$, and are regarded as the design variables for the optimization problem. Besides, the following weighted integrals of the absolute values

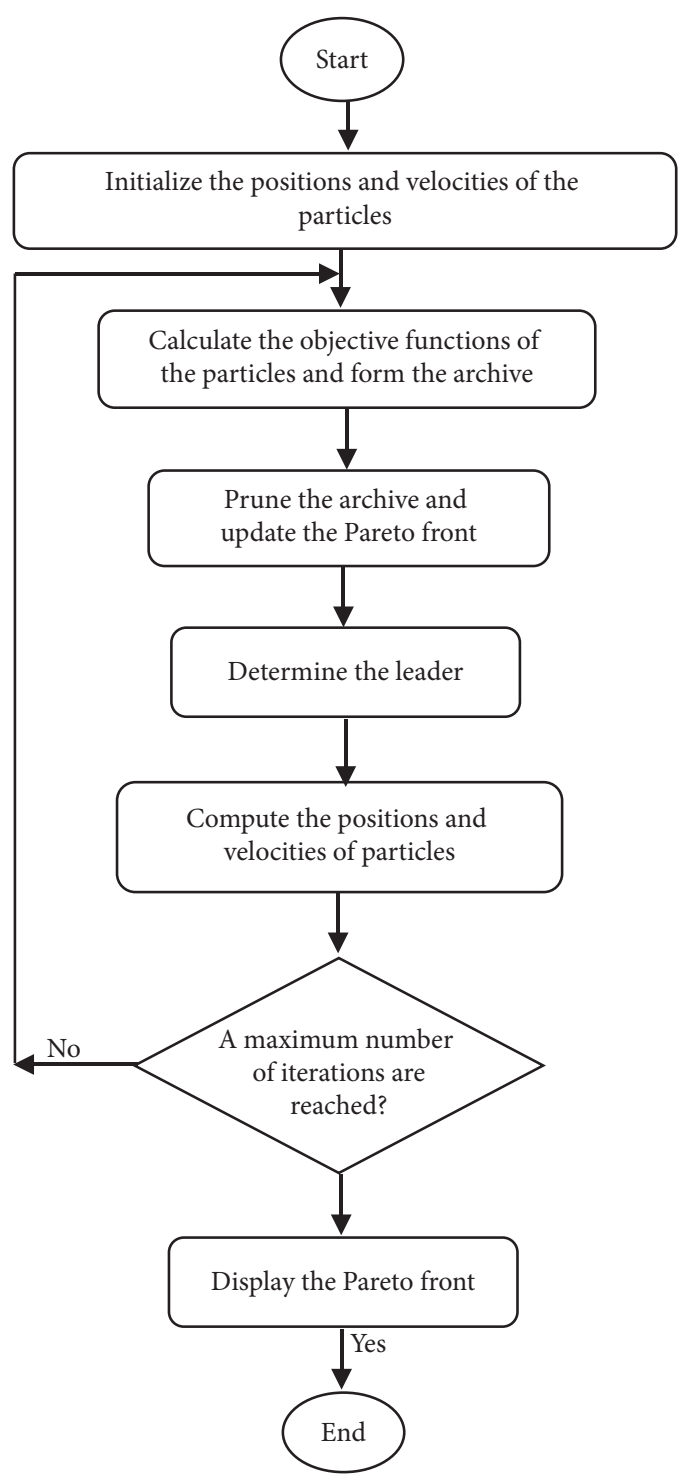

Figure 3: Flowchart of the considered multiobjective optimization algorithm.

of the state error and the control effort are remarked as two objective functions that should be simultaneously minimized:

$$
\begin{aligned}
\text { first objective function (error) } & =w_{1} \int|e| \mathrm{d} t, \\
\text { second objective function (control effort) } & =w_{2} \int|u| \mathrm{d} t,
\end{aligned}
$$

where $w_{1}$ and $w_{2}$ are, respectively, set at 1000 and 0.001 to close the changing ranges of the objective functions.

\section{Results and Discussion}

In this section, the proposed controller is employed for the regarded flexible manipulator having the physical parameters mentioned in Table 1 to track the defined trajectory. The initial conditions are selected as $Z(0)=[(\pi / 6), 0,0,0]$, and the desired trajectory is remarked as $Z_{d}(t)=\sin (t)$. 
TAвLE 1: Physical specifications of the flexible robot manipulator.

\begin{tabular}{lcc}
\hline Parameters & Values & Dimensions \\
\hline$M$ & 4.43 & $\mathrm{~kg}$ \\
$g$ & 9.81 & $\mathrm{~m} / \mathrm{s}^{2}$ \\
$I$ & 1.625 & $\mathrm{~kg} \cdot \mathrm{m}^{2}$ \\
$J$ & 1.625 & $\mathrm{~kg} \cdot \mathrm{m}^{2}$ \\
$L$ & 0.5 & $\mathrm{~m}$ \\
$K$ & 0.5868 & $\mathrm{~kg} / \mathrm{s}^{2}$ \\
\hline
\end{tabular}

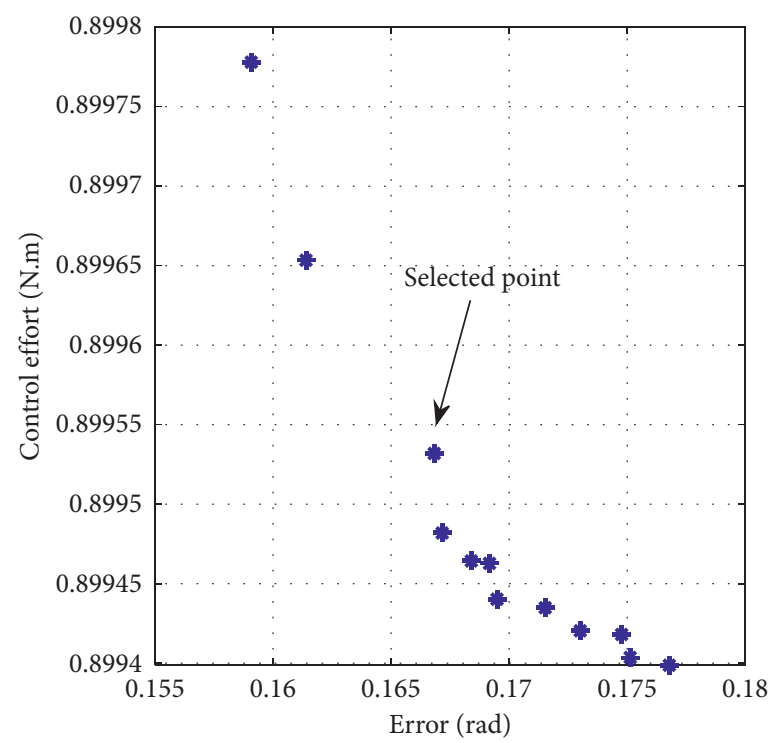

Figure 4: Pareto front obtained by the multiobjective algorithm related to the proposed fuzzy adaptive sliding mode-based feedback linearization controller.

TABLE 2: Optimum values of the parameters in the adaptation laws.

\begin{tabular}{lc}
\hline Parameters & Values \\
\hline$\delta$ & 1.976908 \\
$\sigma$ & 6.115976 \\
$\varnothing$ & 0.434124 \\
$\widehat{\delta}(0)$ & 0.525653 \\
$\widehat{\sigma}(0)$ & 6.314777 \\
$\hat{\varnothing}(0)$ & 4.769374 \\
\hline
\end{tabular}

TABLE 3: Optimum values of the constant parameters related to the fuzzy rule base.

\begin{tabular}{lc}
\hline Parameters & Values \\
\hline$B_{1}^{1}$ & 5.090317 \\
$B_{1}^{2}$ & -3.92817 \\
$B_{1}^{3}$ & 0.979715 \\
$B_{2}^{1}$ & 0.871312 \\
$B_{2}^{2}$ & -6.19309 \\
$B_{2}^{3}$ & -0.50599 \\
$B_{3}^{1}$ & -5.12975 \\
$B_{3}^{2}$ & 6.215155 \\
$B_{3}^{3}$ & -3.09964 \\
\hline
\end{tabular}

In the multiobjective optimization process, the function evaluation of the objective functions is set at 3000. The Pareto front found using the proposed multiobjective particle swarm optimization algorithm is depicted in Figure 4. As it was mentioned before this, all optimum points in this diagram are nondominated to each other, and a designer could choose one of them based on the design criterion. The optimum point selected in this work is shown in the Pareto front of Figure 4, and the related design variables are demonstrated in Tables 2 and 3 for the adaptation laws and fuzzy rules, respectively. Moreover, changing of the fuzzy parameters $\left(\gamma_{1}, \gamma_{2}\right.$, and $\left.\gamma_{3}\right)$, the tuned parameters $(\widetilde{\sigma}, \widetilde{\delta}$, and $\widetilde{\varnothing})$, and the adaptive parameters $(\widehat{\sigma}, \widehat{\delta}$, and $\widehat{\varnothing})$ is displayed in Figures 5-7, respectively. As it can be observed from these figures, all sets of the variables converge to the constant values after the transient time about $2 \mathrm{~s}$. Figures 8-11 depict the time histories of the joint angle, angular velocity, control effort, and phase plane diagrams, respectively. In these figures, the results obtained from the optimized fuzzy adaptive sliding modebased feedback linearization controller are compared with those of the feedback linearization-based proportional derivative (FLPD) method introduced in [53]. As it could be evident from these diagrams, the approach 

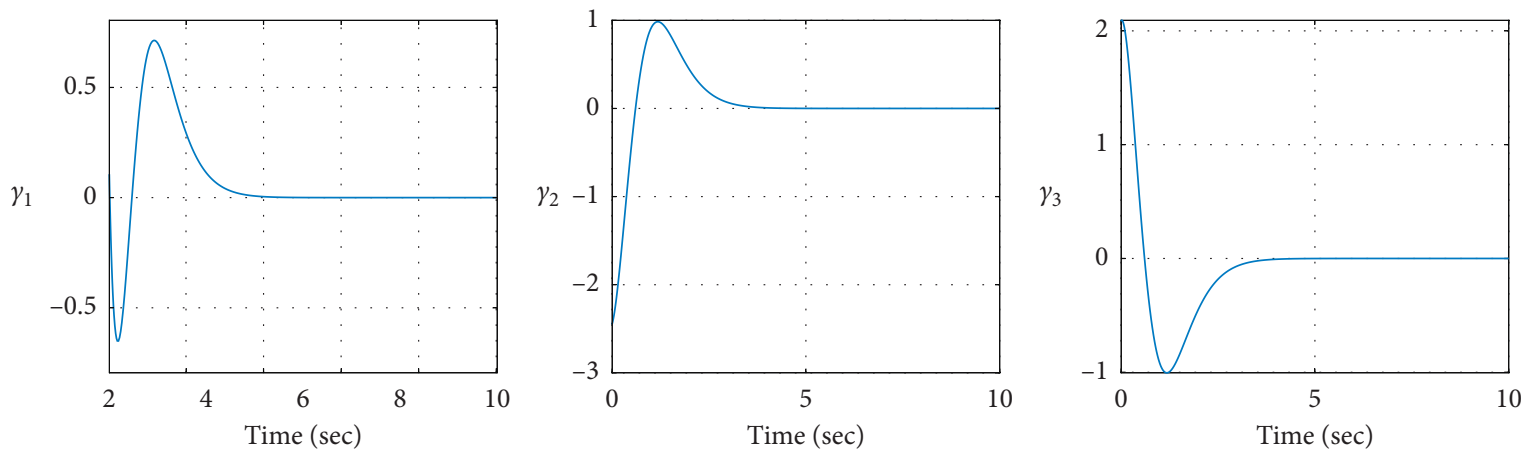

Figure 5: Changing of the fuzzy parameters $\left(\gamma_{1}, \gamma_{2}\right.$, and $\left.\gamma_{3}\right)$ over time.
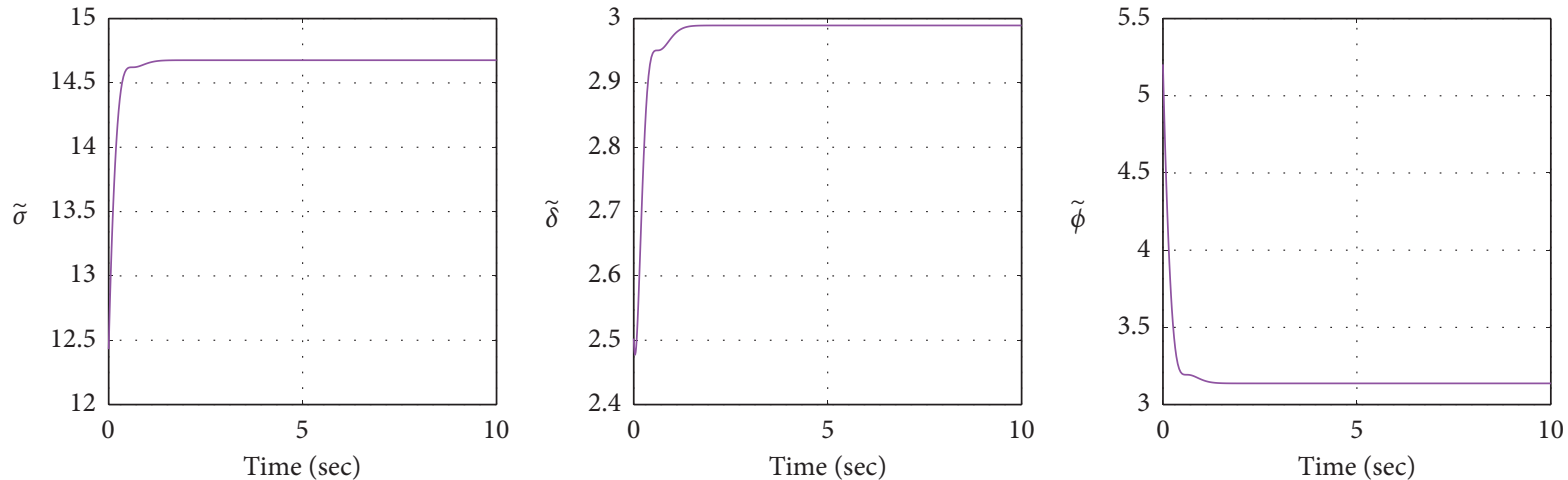

Figure 6: Changing of the tuned parameters $(\widetilde{\sigma}, \widetilde{\delta}$, and $\widetilde{\varnothing})$ over time.
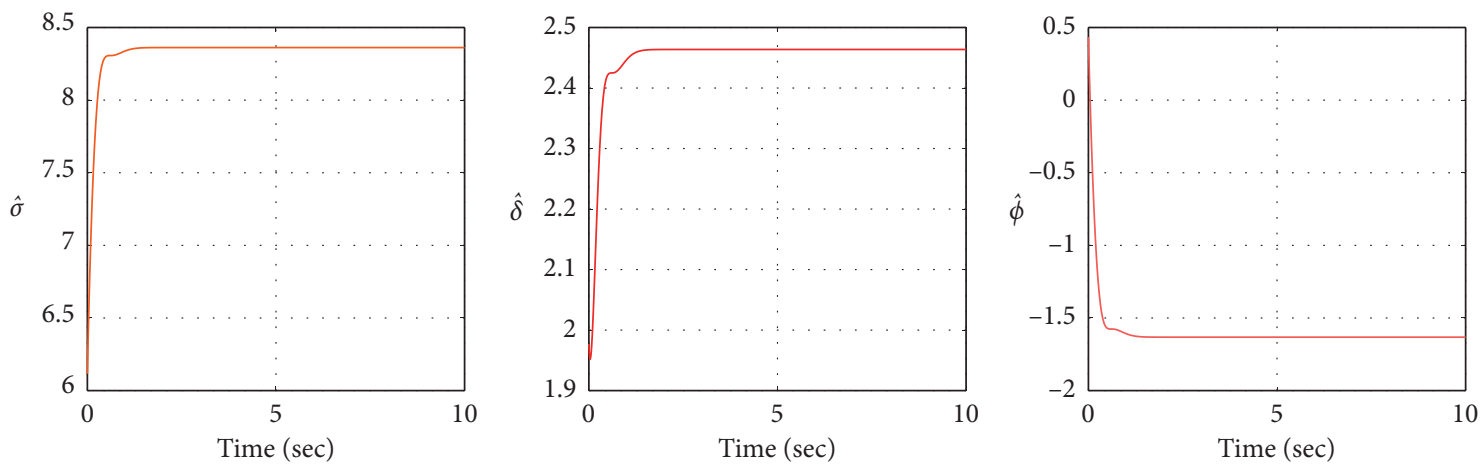

FIGURE 7: Changing of the adaptive parameters $(\widehat{\sigma}, \widehat{\delta}$, and $\widehat{\varnothing})$ over time.

proposed by this work forces the robot to track the desired trajectory in about $2 \mathrm{~s}$, while the feedback linearization-based proportional derivative achieves it in about $5 \mathrm{~s}$.
Generally, the suggested strategy in this research work is able to converge the joint angles and angular velocities to the desired trajectories with less errors in the shorter settling time in comparison with the FLPD controller. However, the 


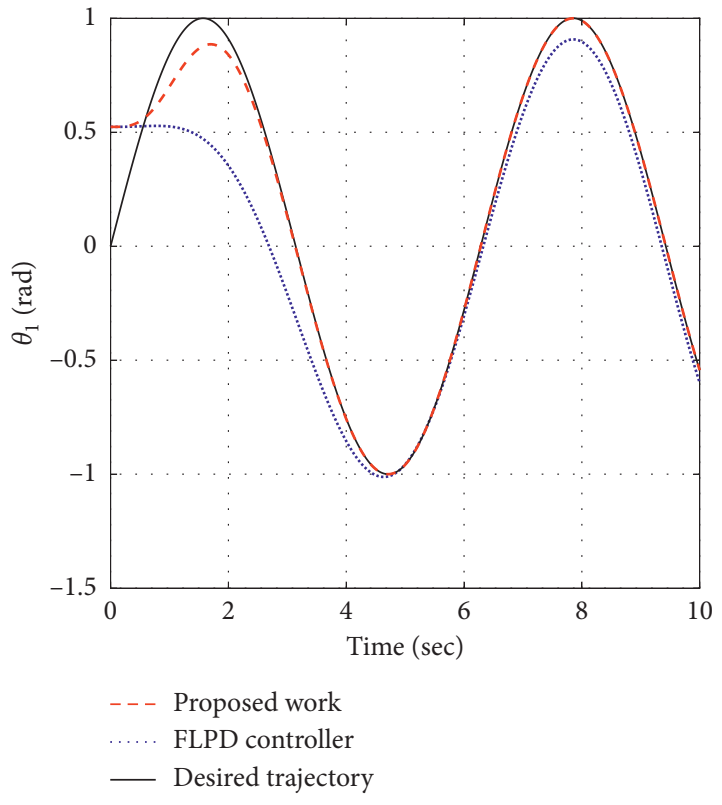

FIGURE 8: Trajectory tracking of the joint angle for the flexible link.

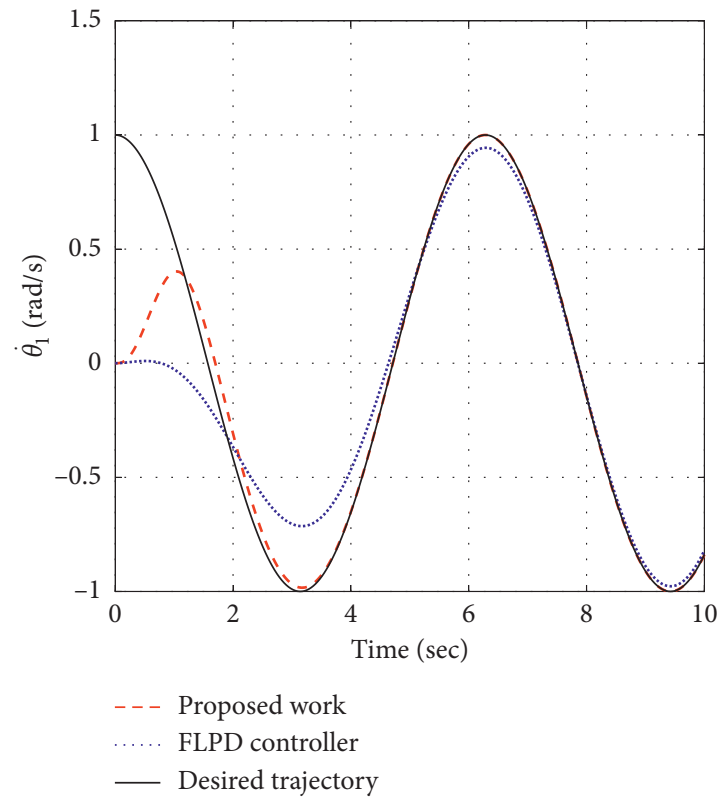

FIGURE 9: Trajectory tracking of the joint angular velocity for the flexible link.

fuzzy adaptive sliding mode-based feedback linearization approach utilizes a bit more control effort compared with the FLPD scheme.

\section{Conclusions and Future Work}

A novel control method as a combination of the feedback linearization scheme, sliding mode control, adaptation laws, and fuzzy systems has been introduced in this work. At first, the feedback linearization method has been successfully utilized to change the nonlinear states of the system to their linear forms. Next, a robust sliding mode control approach

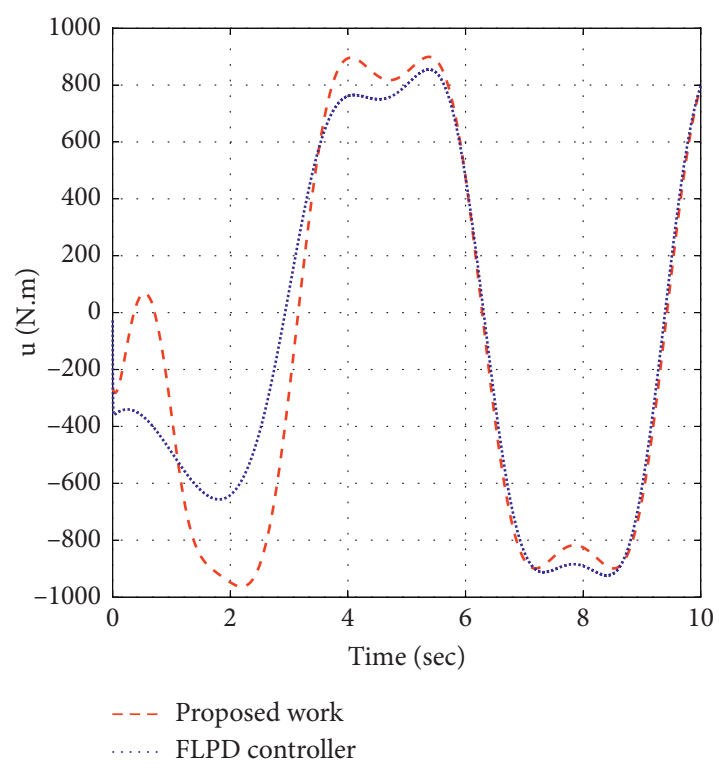

FIgURE 10: Control effort diagram for the flexible link.

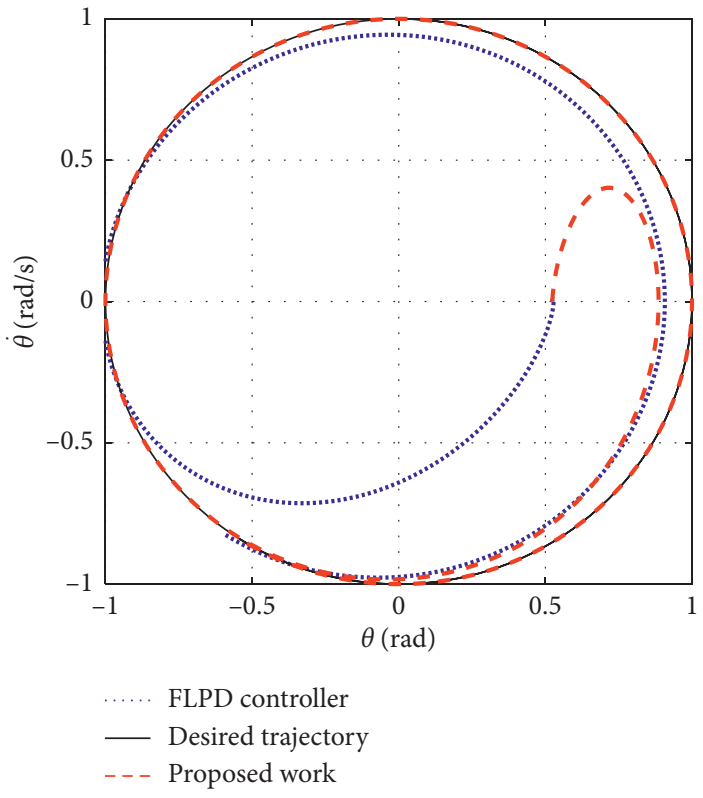

FIGURE 11: Phase plane diagram for the flexible link.

has been implemented to find the related control effort to track the desired trajectory designed for a robot manipulator with the flexible link. In order to timely adapt the control parameters, a combination of the gradient descent methodology and the sliding mode surface has been utilized. A Takagi-Sugeno-Kang fuzzy system has been employed to regulate the constant parameters of the control system. This problem has been considered as a multiobjective optimization problem and solved using the high exploration swarm optimization. The obtained Pareto front has been illustrated, and one of the depicted nondominated solutions has been selected to simulate the control performance of the regarded 
flexible-link robot. The efficiency and ability of the introduced scenario have been easily shown via the diagrams of the state variables compared with other convenient methods.

The future works related to this research could be mentioned as follows:

(1) The suggested scenario is used to control robots with more degrees of freedom

(2) The integral sliding mode surfaces are utilized to design the controller and adaptation laws

(3) Parametric and structural uncertainties are added to the dynamical system to challenge the performance of the proposed stabilizer

(4) Neural networks and moving least squares are applied to timely tune the control gains against the time-varying uncertainties

(5) The introduced optimum fuzzy adaptive robustbased feedback linearization controller is employed to solve actual problems in the real-world application

\section{Data Availability}

No data were used to support the findings of this study.

\section{Conflicts of Interest}

The authors declare that they have no conflicts of interest.

\section{References}

[1] C. A. My, D. X. Bien, C. H. Le, and M. Packianather, “An efficient finite element formulation of dynamics for a flexible robot with different type of joints," Mechanism and Machine Theory, vol. 134, pp. 267-288, 2019.

[2] L. Sun, W. Zhao, W. Yin, N. Sun, and J. Liu, "Proxy based position control for flexible joint robot with link side energy feedback," Robotics and Autonomous Systems, vol. 121, Article ID 103272, 2019.

[3] K. Dong, H. Liu, X. Zhu, X. Wang, F. Xu, and B. Liang, "Forcefree control for the flexible-joint robot in human-robot interaction," Computers \& Electrical Engineering, vol. 73, pp. 9-22, 2019.

[4] D. Feliu-Talegon, V. Feliu-Batlle, I. Tejado, B. M. Vinagre, and S. H. HosseinNia, "Stable force control and contact transition of a single link flexible robot using a fractional-order controller," ISA Transactions, vol. 89, pp. 139-157, 2019.

[5] O. A. Garcia-Perez, G. Silva-Navarro, and J. F. Peza-Solis, "Flexible-link robots with combined trajectory tracking and vibration control," Applied Mathematical Modelling, vol. 70, pp. 285-298, 2019.

[6] P. Li, L. Xiong, F. Wu, M. Ma, and J. Wang, "Sliding mode controller based on feedback linearization for damping of sub-synchronous control interaction in DFIG-based wind power plants," International Journal of Electrical Power \& Energy Systems, vol. 107, pp. 239-250, 2019.

[7] A. Ammar, A. Kheldoun, B. Metidji, T. Ameid, and Y. Azzoug, "Feedback linearization based sensorless direct torque control using stator flux MRAS-sliding mode observer for induction motor drive," ISA Transactions, vol. 98, pp. 382-392, 2020.

[8] J. C. Cambera and V. Feliu-Batlle, "Input-state feedback linearization control of a single-link flexible robot arm moving under gravity and joint friction," Robotics and $\mathrm{Au}$ tonomous Systems, vol. 88, pp. 24-36, 2017.

[9] M. Ruderman, "Feedback linearization control of flexible structures with hysteresis," IFAC PapersOnLine, vol. 48, no. 11, pp. 906-911, 2015.

[10] S. Moberg and S. Hanssen, "On feedback linearization for robust tracking control of flexible joint robots," IFAC Proceedings Volumes, vol. 41, no. 2, pp. 12218-12223, 2008.

[11] S.-J. Huang, H.-Y. Chen, Variable structure systems with sliding modes," IEEE Transactions of Automatic Control, vol. 2, pp. 212-222, 1977.

[12] Y. Xiong, Y. Gao, L. Yang, and L. Wu, “An integral sliding mode approach to distributed control of coupled networks with measurement Quantization," Systems \& Control Letters, vol. 133, Article ID 104557, 2019.

[13] Z. Yuguang and Y. Fan, "Dynamic modeling and adaptive fuzzy sliding mode control for multi-link underwater manipulators," Ocean Engineering, vol. 187, Article ID 106202, 2019.

[14] S. Wang, Q. Chen, X. Ren, and H. Yu, "Neural network-based adaptive funnel sliding mode control for servo mechanisms with friction compensation," Neurocomputing, vol. 377, pp. 16-26, 2020.

[15] M. Divandari, B. Rezaie, and A. Ranjbar Noei, "Speed control of switched reluctance motor via fuzzy fast terminal slidingmode control," Computers \& Electrical Engineering, vol. 80, Article ID 106472, 2019.

[16] S. Zaare, M. R. Soltanpour, and M. Moattari, "Voltage based sliding mode control of flexible joint robot manipulators in presence of uncertainties," Robotics and Autonomous Systems, vol. 118, pp. 204-219, 2019.

[17] J. F. Peza-Solís, G. Silva-Navarro, and N. R. Castro-Linares, "Trajectory tracking control in a single flexible-link robot using finite differences and sliding modes," Journal of Applied Research and Technology, vol. 13, no. 1, pp. 70-78, 2015.

[18] D. Hisseine and B. Lohmann, "Sliding mode tracking control for a single-link flexible robot arm," IFAC Proceedings Volumes, vol. 33, no. 27, pp. 333-338, 2000.

[19] Y. Miao, I. Hwang, M. Liu, and F. Wang, "Adaptive fast nonsingular terminal sliding mode control for attitude tracking of flexible spacecraft with rotating appendage," Aerospace Science and Technology, vol. 93, Article ID 105312, 2019.

[20] Z. Zhao, X. He, and G. Wen, "Boundary robust adaptive antisaturation control of vibrating flexible riser systems," Ocean Engineering, vol. 179, pp. 298-306, 2019.

[21] S. Boulouma, S. Labiod, and H. Boubertakh, "Direct adaptive control of a flexible spacecraft with disturbances and uncertain actuator failures," Mechanical Systems and Signal Processing, vol. 110, pp. 73-89, 2018.

[22] P. Gierlak and M. Szuster, "Adaptive position/force control for robot manipulator in contact with a flexible environment," Robotics and Autonomous Systems, vol. 95, pp. 80-101, 2017.

[23] A. Seyed Danesh, M. Dadkhah, N. Misaghian, and N. D. Pavlović, "Adaptive control algorithm of flexible robotic gripper by extreme learning machine," Robotics and Computer-Integrated Manufacturing, vol. 37, pp. 170-178, 2016.

[24] K. Zheng, Y. Hu, and B. Wu, "Intelligent fuzzy sliding mode control for complex robot system with disturbances," European Journal of Control, vol. 51, pp. 95-109, 2020.

[25] W. Shi, "Observer-based adaptive fuzzy prescribed performance control for feedback linearizable MIMO nonlinear systems with unknown control direction," Neurocomputing, vol. 368, pp. 99-113, 2019. 
[26] L. Hongqian, H. Xianlin, G. XZ, B. Xiaojun, and Y. Hang, "Stability analysis of the simplest Takagi-Sugeno fuzzy control system using circle criterion," Journal of Systems Engineering and Electronics, vol. 18, no. 2, pp. 311-319, 2007.

[27] E. Yazid, M. Garratt, and F. Santoso, "Position control of a quadcopter drone using evolutionary algorithms-based selftuning for first-order Takagi-Sugeno-Kang fuzzy logic autopilots," Applied Soft Computing, vol. 78, pp. 373-392, 2019.

[28] R. Laina, F. Ez-Zahra Lamzouri, E.-M. Boufounas, A. El Amrani, and I. Boumhidi, "Intelligent control of a DFIG wind turbine using a PSO evolutionary algorithm," Procedia Computer Science, vol. 127, pp. 471-480, 2018.

[29] M. H. Oboudi, R. Hooshmand, and A. Karamad, "A feasible method for controlled intentional islanding in microgrids based on PSO algorithm," Swarm and Evolutionary Computation, vol. 35, pp. 14-25, 2017.

[30] L. Cao, D. Zhang, S. Tang, and F. Deng, "A practical parameter determination strategy based on improved hybrid PSO algorithm for higher-order sliding mode control of airbreathing hypersonic vehicles," Aerospace Science and Technology, vol. 59, pp. 1-10, 2016.

[31] B. Naidu Kommula and V. Reddy Kota, "Direct instantaneous torque control of Brushless DC motor using firefly Algorithm based fractional order PID controller," Journal of King Saud University-Engineering Sciences, vol. 32, no. 2, pp. 133-140, 2020.

[32] F. Yan, "Autonomous vehicle routing problem solution based on artificial potential field with parallel ant colony optimization (ACO) algorithm," Pattern Recognition Letters, vol. 116, no. 1, pp. 195-199, 2018.

[33] A. Gonzalez-Pardo, J. Del Ser, and D. Camacho, "Comparative study of pheromone control heuristics in ACO algorithms for solving RCPSP problems," Applied Soft Computing, vol. 60, pp. 241-255, 2017.

[34] H. Li, S. Liu, M. M. Hassan, S. Ali, and Z. Xu, "Rapid quantitative analysis of $\mathrm{Hg} 2+$ residue in dairy products using SERS coupled with ACO-BP-AdaBoost algorithm," Spectrochimica Acta Part A: Molecular and Biomolecular Spectroscopy, vol. 223, no. 5, Article ID 117281, 2019.

[35] M. J. Mahmoodabadi and D. Roshandel, "An optimal fuzzy controller based on the inverse dynamic approach using real ant colony optimization for nonlinear robot manipulators," International Journal of Mechanics and Control, vol. 20, pp. 93-104, 2019.

[36] B. Singh, V. Mukherjee, and P. Tiwari, "GA-based optimization for optimally placed and properly coordinated control of distributed generations and Static Var Compensator in distribution networks," Energy Reports, vol. 5, pp. 926-959, 2019.

[37] Y. Shin, Z. Kim, J. Yu, G. Kim, and S. Hwang, "Development of NOx reduction system utilizing artificial neural network (ANN) and genetic algorithm (GA)," Journal of Cleaner Production, vol. 232, pp. 1418-1429, 2019.

[38] K. Liu and B. Liu, "Optimization of smooth blasting parameters for mountain tunnel construction with specified control indices based on a GA and ISVR coupling algorithm," Tunnelling and Underground Space Technology, vol. 70, pp. 363-374, 2017.

[39] H. Bakir and A. Afsin Kulaksiz, "Modelling and voltage control of the solar-wind hybrid micro-grid with optimized STATCOM using GA and BFA," Engineering Science and Technology, An International Journal, vol. 23, no. 3, pp. 576-584, 2020.
[40] A. Vieira, R. Marques, R. Raposo et al., "The impact of the artICA control technology on the performance, energy consumption and greenhouse gas emissions of full-scale wastewater treatment plants," Journal of Cleaner Production, vol. 213, pp. 680-687, 2019.

[41] M. S. Salman, Y. Du, D. Lin, Z. Fu, and V. D. Calhoun, "Group ICA for identifying biomarkers in schizophrenia: "Adaptive" networks via spatially constrained ICA show more sensitivity to group differences than spatio-temporal regression," NeuroImage: Clinical, vol. 22, Article ID 101747, 2019.

[42] N. A. Reisi, S. Hadipour Lakmesari, M. J. Mahmoodabadi, and S. Hadipour, "Optimum fuzzy control of human immunodeficiency virus typel using an imperialist competitive algorithm," Informatics in Medicine Unlocked, vol. 16, Article ID 100241, 2019.

[43] M. J. Mahmoodabadi, M. Rasekh, and T. Zohari, "TGA: team game algorithm," Future Computing and Informatics Journal, vol. 3, no. 2, pp. 191-199, 2018.

[44] M. R. Khalghani and M. H. Khooban, "A novel self-tuning control method based on regulated bi-objective emotional learning controller's structure with TLBO algorithm to control DVR compensator," Applied Soft Computing, vol. 24, pp. 912-922, 2014.

[45] M. J. Mahmoodabadi and R. Ostadzadeh, "CTLBO: Converged teaching-learning-based optimization," Cogent Engineering, vol. 6, no. 1, Article ID 1654207, 2019.

[46] K. Abd, K. Abhary, and R. Marian, "Multi-objective optimisation of dynamic scheduling in robotic flexible assembly cells via fuzzy-based Taguchi approach," Computers \& Industrial Engineering, vol. 99, pp. 250-259, 2016.

[47] X. Liu and J. Luo, "A dynamic multi-objective optimization model with interactivity and uncertainty for real-time reservoir flood control operation," Applied Mathematical Modelling, vol. 74, pp. 606-620, 2019.

[48] P. Wang, X. Yan, and F. Zhao, "Multi-objective optimization of control parameters for a pressurized water reactor pressurizer using a genetic algorithm," Annals of Nuclear Energy, vol. 124, pp. 9-20, 2019.

[49] L. Chen, Q. Li, X. Zhao, Z. Fang, F. Peng, and J. Wang, "Multipopulation coevolutionary dynamic multi-objective particle swarm optimization algorithm for power control based on improved crowding distance archive management in CRNs," Computer Communications, vol. 145, pp. 146-160, 2019.

[50] M. Kaucic, "Equity portfolio management with cardinality constraints and risk parity control using multi-objective particle swarm optimization," Computers \& Operations Research, vol. 109, pp. 300-316, 2019.

[51] A. Farokhi and M. J. Mahmoodabadi, "Optimal fuzzy inverse dynamics control of a parallelogram mechanism based on a new multi-objective PSO," Cogent Engineering, vol. 5, no. 1, 2018.

[52] M. J. Mahmoodabadi, Z. Salahshoor Mottaghi, and A. Bagheri, "HEPSO: high exploration particle swarm optimization," Information Sciences, vol. 273, pp. 101-111, 2014.

[53] M. W. Spong, S. Hutchinson, and M. Vidyasagar, Robot Dynamics and Control, 2004.

[54] J. X. Xu and W. Cao, "Synthesized sliding mode control of a single-link flexible robot," IFAC Proceedings Volumes, vol. 32, no. 2, pp. 3277-3282, 1999.

[55] A. Ficola, M. La Cava, and P. Muraca, "A simplified strategy to implement sliding mode control of a two-joints robot with a flexible forearm," Motion Control for Intelligent Automation, vol. 25, no. 29, pp. 283-288, 1992. 
[56] H. M. Yen, T. H. S. Li, and Y. C. Chang, "Adaptive neural network based tracking control for electrically driven flexiblejoint robots without velocity measurements," Computers \& Mathematics with Applications, vol. 64, no. 5, pp. 1022-1032, 2012.

[57] Y. Li, S. Tong, and T. Li, "Adaptive fuzzy output feedback control for a single-link flexible robot manipulator driven DC motor via backstepping," Nonlinear Analysis: Real World Applications, vol. 14, no. 1, pp. 483-494, 2013.

[58] A. Green and J. Z. Sasiadek, "Repetitive learning with fuzzy logic adaptive control of a flexible robot manipulator," IFAC Proceedings Volumes, vol. 36, no. 17, pp. 569-574, 2003.

[59] C. Baspinar, "Position control of flexible joint robots by adapting methods for rigid robots," IFAC Proceedings Volumes, vol. 44, no. 1, pp. 13498-13503, 2011.

[60] J. I. Arciniegas, A. H. Eltimsahy, and K. J. Cios, "Neuralnetworks-based adaptive control of flexible robotic arms," Neurocomputing, vol. 17, no. 3-4, pp. 141-157, 1997.

[61] M. Jafarzadeh, N. Gans, and Y. Tadesse, "Control of TCP muscles using Takagi-Sugeno-Kang fuzzy inference system," Mechatronics, vol. 53, pp. 124-139, 2018.

[62] S.-Y. Wang, F.-Y. Liu, and J.-H. Chou, "Adaptive TSK fuzzy sliding mode control design for switched reluctance motor DTC drive systems with torque sensorless strategy," Applied Soft Computing, vol. 66, pp. 278-291, 2018.

[63] V. Feliu, E. Pereira, and I. M. Díaz, "Passivity-based control of single-link flexible manipulators using a linear strain feedback," Mechanism and Machine Theory, vol. 71, pp. 191-208, 2014.

[64] O. Shaheen, A. M. El-Nagar, M. El-Bardini, and N. M. ElRabaie, "Stable adaptive probabilistic Takagi-Sugeno-Kang fuzzy controller for dynamic systems with uncertainties," ISA Transactions, vol. 98, pp. 271-283, 2020.

[65] R.-E. Precup, H.-I. Filip, M.-B. Rădac, E. M. Petriu, S. Preitl, and C.-A. Dragoş, "Online identification of evolving TakagiSugeno-Kang fuzzy models for crane systems," Applied Soft Computing, vol. 24, pp. 1155-1163, 2014.

[66] J. Kennedy and R. Eberhart, "Particle swarm optimization," in Proceedings of ICNN'95, International Conference on Neural Networks, Perth, WA, Australia, 1995. 